\title{
Cross-genera SSR transferability in cacti revealed by a case study using Cereus (Cereeae, Cactaceae)
}

Juliana Rodrigues Bombonato ${ }^{1,2}$ (D), Isabel Aparecida Silva Bonatelli ${ }^{1}$ (D), Gislaine Angélica Rodrigues Silva $^{1}$ (D), Evandro Marsola Moraes ${ }^{1}$ (D), Daniela Cristina Zappi ${ }^{3}$ (D), Nigel P. Taylor ${ }^{4}$ and Fernando Faria Franco $^{1}$ iD

${ }^{1}$ Departamento de Biologia, Centro de Ciências Humanas e Biológicas, Universidade Federal de São Carlos, Sorocaba, SP, Brazil.

${ }^{2}$ Programa de Pós-graduação em Biologia Comparada, Faculdade de Filosofia, Ciências e Letras de Ribeirão Preto, Universidade de São Paulo, Ribeirão Preto, SP, Brazil.

${ }_{3}^{3}$ Instituto Tecnológico Vale, Museu Paraense Emilio Goeldi, Coord. Botânica, Belém, PA, Brazil.

${ }^{4}$ Singapore Botanic Gardens (National Parks Board), Singapore, Republic of Singapore.

\begin{abstract}
The study of transferability of simple sequence repeats (SSR) among closely related species is a well-known strategy in population genetics, however transferability among distinct genera is less common. We tested cross-genera SSR amplification in the family Cactaceae using a total of 20 heterologous primers previously developed for the genera Ariocarpus, Echinocactus, Polaskia and Pilosocereus, in four taxa of the genus Cereus: C. fernambucensis subsp. fernambucensis, $C$. fernambucensis subsp. sericifer, $C$. jamacaru and $C$. insularis. Nine microsatellite loci were amplified in Cereus resulting in $35.2 \%$ of success in transferability, which is higher than the average rate of $10 \%$ reported in the literature for cross-genera transferability in eudicots. The genetic variation in the transferred markers was sufficient to perform standard clustering analysis, indicating each population as a cohesive genetic cluster. Overall, the amount of genetic variation found indicates that the transferred SSR markers might be useful in large-scale population studies within the genus Cereus.
\end{abstract}

Keywords: Cactaceae, Cereus, cross-genera, SSR markers, Transferability.

Received: September 13, 2017; Accepted: June 13, 2018.

Simple sequence repeats (SSR) or microsatellites are, in general, non-coding regions commonly found in Eukaryote genomes composed of tandemly arranged repeat motifs from 1 to 6 base pairs (Oliveira et al., 2006). SSRs are useful molecular markers for several applications in population genetics and breeding studies, as they frequently exhibit high levels of polymorphism, in addition to their abundance and random distribution across and throughout genomes. In plants, SSR loci have been used for several purposes, for example, estimates of genetic diversity (Zhu et al., 2016), intra- and interspecific gene flow (PalmaSilva et al., 2011; Pinheiro et al., 2014), biogeographical distributions (Beatty and Provan 2011), phylogenetic relationships (Mehmood et al., 2016), genetic mapping (Tan et al., 2016), and conservation (Gómez-Fernández et al., 2016).

Send correspondence to Fernando Faria Franco, Departamento de Biologia, Centro de Ciências Humanas e Biológicas, Universidade Federal de São Carlos, Rodovia João Leme dos Santos, SP264 $\mathrm{Km} \mathrm{110,} \mathrm{18052-780,} \mathrm{Sorocaba,} \mathrm{SP,} \mathrm{Brazil.} \mathrm{E-mail:}$ franco@ufscar.br.
An alternative to overcome time consuming and costly development of a new set of SSR primers for a target species is to carry out the transferability of SSR primers among related species (Barbará et al., 2007; Lavor et al., 2013; Nogueira et al., 2015). The rate of success in this approach (i.e., heterologous amplification) depends on the nucleotide similarity among the flanking regions of different species. Therefore, it is expected that there will be a higher rate in heterologous amplification among taxa with recent divergence times. In plants, this technique has been widely adopted for a great variety of eudicots (e.g., Haerinasab et al., 2016; Mengistu et al., 2016), where the average rate of success at infrageneric level is around $60 \%$ (Barbará et al., 2007). The rate of cross-genera transferability is around $10 \%$ in eudicots (Barbará et al., 2007), but the levels of success may reach values above $50 \%$ in some plants (Satya et al., 2016).

Taking into account the recent divergence within Cactaceae, as well as its emergence as an informative model to study diversification in xeric habitats (Arakaki et al., 2011), the aim of this study was to perform cross-genera 
SSR amplification in four closely related taxa of the genus Cereus (Cactaceae; Cereeae) occurring in eastern Brazil: $C$. fernambucensis subsp. fernambucensis, C. fernambucensis subsp. sericifer, C. insularis and C. jamacaru. A previous phylogenetic analysis based on plastid DNA placed $C$. jamacaru as a member of a polytomic clade, sister of the monophyletic clade composed by $C$. fernambucensis and C. insularis (Franco et al., 2017a). In this study, we selected a set of 11 SSR loci originally described for Ariocarpus bravoanus (Hughes et al., 2008), Echinocactus grusonii (Hardesty et al., 2008) and Polaskia chichipe (Otero-Arnaiz et al., 2004) that were recently transferred to Cereus species cultivated in different urban areas, including C. hildmannianus (Martin 2011; Fernandes et al., 2016). An additional nine SSR loci described for Pilosocereus machrisii (Perez et al., 2011) were included in this investigation.

We sampled 122 individuals from representative populations of $C$. jamacaru, $C$. insularis and $C$. fernambucensis (Table 1), besides one individual of $C$. hildmannianus (Salto, SP; 23.99, 47.33; SORO 2746) as a positive control in the initial tests. Genomic DNA was extracted from the radicular tissue using the Qiagen DNeasy Plant Mini Kit (Qiagen, Hilden, Germany). As the samples from localities S82 and S83 are geographically $(\sim 34 \mathrm{~km})$ and genetically close, sharing the same unique alleles and comprising a cohesive genetic group, we decided to join the individuals from the two populations in a single sample, hereinafter referred to as $\mathrm{S} 82 / \mathrm{S} 83$.

Initial amplification tests were performed using a subsample of 12 individuals, with slight modifications on PCR conditions as described by Albert and Schmitz (2002), Don et al. (1991), and Perez et al. (2011). The reactions were performed in $10 \mu \mathrm{L}$ of total PCR volume including 0.5 U of Taq DNA Polymerase (Promega), 1X Taq Buffer (5X
Colorless GoTaq ${ }^{\circledR}$ Flexi Buffer), $0.2 \mu \mathrm{M}$ dNTPs, and primer and $\mathrm{MgCl}_{2}$ concentrations varying when necessary. We considered a locus successfully transferred when the PCR products were clearly visualized in 3\% agarose gels and showed a product size compatible with the range described for that locus. The loci successfully amplified were then genotyped in the total sample (Table 1) using PAGE (denaturing polyacrylamide gel) with concentrations varying between $6 \%$ to $9 \%$, according to expected allele size. To visualize the alleles, the gels were stained with silver nitrate. The percentage of transferability success was estimated according to the number of individuals amplified in each locus.

The occurrence of null alleles, allele drop-out, and stutter bands was evaluated with Micro-Checker 2.2.3 software (Van Oosterhout et al., 2004). The number of alleles per locus $\left(N_{\mathrm{a}}\right)$, effective number of alleles $\left(n_{\mathrm{e}}\right)$, expected $\left(H_{\mathrm{e}}\right)$ and observed $\left(H_{\mathrm{o}}\right)$ heterozygosities, private alleles, and percentage of polymorphic loci were estimated using GenAlEx 6.5 software (Peakall and Smouse, 2012). The inbreeding coefficient $\left(F_{\text {IS }}\right)$ per population was calculated using FSTAT 2.9.3.2 (Goudet, 1995), assuming $\alpha=0.01$ and $\alpha=0.001$ (Lavor et al., 2013; Ribeiro et al., 2014). Deviations from Hardy-Weinberg equilibrium (HWE) and linkage disequilibrium (LD) were investigated using the Arlequin 3.5.1.3 program (Excoffier and Lischer, 2010). We used the sequential Bonferroni correction for multiple testing with $\alpha=0.05$ (Rice, 1989) to minimize statistical errors. Genetic differentiation among populations was quantified by $F_{\mathrm{ST}}$ (Weir and Cockerham, 1984) estimated in FSTAT 2. 9. 3.2 (Goudet, 1995) and corrected for null alleles in FreeNA (Chapuis and Estoup, 2007). The pairwise chord distances (Dc, Cavalli-Sforza and Edwards, 1967) between populations was estimated in FreeNA software (Chapuis and Estoup, 2007), and the resulting matrix was

Table 1 - Geographical localities of the populations from three species of Cereus used in this work.

\begin{tabular}{|c|c|c|c|}
\hline Species & Voucher & Geographic Coordinates $(\mathrm{S}, \mathrm{W})$ & $\mathrm{N}$ \\
\hline \multicolumn{4}{|l|}{ C. fernambucensis subsp. fernambucensis Lem. } \\
\hline Arraial do Cabo, RJ (S80) - Southern group* & SORO 2663 & $-22.97,-42.03$ & 19 \\
\hline Maracajaú, RN (S104) - Northern group* & SORO 4529 & $-5.39,-35.31$ & 20 \\
\hline Una, BA (S114) - Northern group* & SORO 2675 & $-15.11,-39.00$ & 20 \\
\hline \multicolumn{4}{|l|}{ C. fernambucensis subsp. sericifer Ritt. } \\
\hline Santa Maria Madalena, RJ (S82) - Southern group* & SORO 2665 & $-21.95,-42.03$ & 12 \\
\hline Itaoacara, RJ (S83) - Southern group* & SORO 2666 & $-21.65,-42.09$ & 4 \\
\hline Águia Branca, ES (S88) - Northern group* & SORO 2749 & $-19.06,-40.69$ & 10 \\
\hline \multicolumn{4}{|l|}{ C. jamacaru DC. } \\
\hline Conceição de Feira, BA (S113) & HUEFS 33711 & $-12.59,-38.99$ & 18 \\
\hline \multicolumn{4}{|l|}{ C. insularis Hmsl. } \\
\hline Fernando de Noronha, PE (S115D) & SORO 2677 & $-3.85,-32.40$ & 19 \\
\hline
\end{tabular}

* The classification in Southern and Northern group is based on phylogeographic data available for C. fernambucensis (Franco et al., 2017b) / N = number of individuals per populations. 
then used as input to generate a Neighbor-Joining dendrogram (NJ) (Saitou and Nei, 1987) in Populations 1.2.32 software (Langella, 1999). To explore genetic structure in our data we performed: a Principal Coordinate Analysis (PCoA) in GenAlEx 6.5 (Peakall and Smouse, 2012); a global and a hierarchical Analysis of Molecular Variance (AMOVA) in Arlequin 3.5.1.3 (Excoffier and Lischer, 2010); and a Bayesian clustering analysis in STRUCTURE 2.3.4 (Pritchard et al., 2000). The latter was implemented using 10 simultaneous and independent runs with $10^{6}$ generations of MCMC ( $25 \%$ as burn-in). The K-values tested ranged from 1 to 8 . To find the best $\mathrm{K}$ we used $\Delta \mathrm{K}$ statistics (Evanno et al., 2005) in Structure Harvester. The results of the independent runs for the best $\mathrm{K}$ were combined in Clumpp (Jakobsson and Rosenberg, 2007), and were graphically displayed with Distruct (Rosenberg, 2004).

From the 20 tested loci (Table S1), nine ( $m A b R 28$ from A. bravoanus; $m E g R$ 02, $m E g R 76$ and $m E g R 78$ from E. grusonii and Pmac82, Pmac84, Pmac108, Pmac146 and Pmac149 from P. machrisii) showed positive results in transferability for at least one species (Table S2), resulting in $35.16 \%$ of success in transferability. Except for $m E g R$
02 , the allele size for all loci was congruent with the expected size (Table S2). We were not able to amplify five SSR loci previously transferred to Cereus (Pchi 21, Pchi 47, Pchi 54, mAbR 42 and $m A b R$ 77) (Martin, 2011; Fernandes et al., 2016), even after several attempts to modify PCR conditions (Table S3). This result is likely related to nucleotide differences among the flanking regions of the samples used in this work, preventing primer annealing.

The percentage of polymorphic loci ranged from $44.4 \%$ (populations S113 and S82/S83) to $77.8 \%$ (location S80) (Table 2). In contrast with the expectation of reduced levels of genetic diversity at transferred SSR loci (e.g. Goldstein and Pollock, 1997; Jan et al., 2012; Moodley et al., 2015), we found higher levels of polymorphism for some loci (Pmac82 in all populations, $m E g R 78$ in $\mathrm{S} 88$ and $m E g R 02$ in S80) than those reported in the original description (Table 2). No locus showed significant heterozygosity deficiency in relation to expectations of HWE after Bonferroni correction. Inbreeding coefficient estimates $\left(F_{\mathrm{IS}}\right)$ provided no significant result (Table 2). The locus Pmac108 showed high levels of observed heterozygosity in all populations, excepting S82/S83 (Table 2). Private al-

Table 2 - Genetic diversity indices: Number of samples (N), Number of alleles $(\mathrm{Na})$, Effective allele number ( $\mathrm{Ne}$ ), Observed heterozygosity $(\mathrm{Ho})$, Expected heterozygosity $(\mathrm{He})$, absence $(-)$ and presence $(+)$ of null alleles, and $F_{I S}$ values per loci and population are shown.

\begin{tabular}{|c|c|c|c|c|c|c|c|c|}
\hline Locus & $\mathrm{N}$ & $\mathrm{Na}$ & $\mathrm{Ne}$ & $\mathrm{Ho}$ & $\mathrm{He}$ & Null Allele & $F_{I S}$ & $p$-value \\
\hline Population S113 & & & & & & & 0.06 & 0.26 \\
\hline Pmac82 & 18 & 3 & 1.57 & 0.33 & 0.36 & - & & \\
\hline Pmac108 & 13 & 5 & 2.38 & 0.62 & 0.58 & - & & \\
\hline Pmac149 & 18 & 5 & 1.81 & 0.50 & 0.45 & - & & \\
\hline$m E g R 02$ & 16 & 1 & 1.00 & 0.00 & 0.00 & - & & \\
\hline$m A b R 28$ & 16 & 1 & 1.00 & 0.00 & 0.00 & - & & \\
\hline$m E g R 76$ & 18 & 2 & 1.12 & 0.00 & 0.11 & - & & \\
\hline Média & 16.5 & 2.83 & 1.48 & 0.24 & 0.25 & & & \\
\hline Population S80 & & & & & & & 0.08 & 0.13 \\
\hline Pmac82 & 19 & 2 & 1.17 & 0.16 & 0.15 & - & & \\
\hline Pmac84 & 18 & 3 & 2.66 & 0.78 & 0.62 & - & & \\
\hline Pmac108 & 18 & 5 & 2.61 & 0.67 & 0.62 & - & & \\
\hline Pmac146 & 17 & 3 & 2.34 & 0.41 & 0.57 & - & & \\
\hline$m E g R 02$ & 16 & 3 & 1.68 & 0.25 & 0.41 & - & & \\
\hline$m A b R 28$ & 14 & 4 & 1.57 & 0.43 & 0.36 & - & & \\
\hline$m E g R 76$ & 17 & 1 & 1.00 & 0.00 & 0.00 & - & & \\
\hline$m E g R 78$ & 17 & 2 & 1.12 & 0.00 & 0.11 & - & & \\
\hline Média & 17.0 & 2.56 & 1.77 & 0.34 & 0.36 & & & \\
\hline Population S82/S83 & & & & & & & 0.05 & 0.35 \\
\hline Pmac82 & 16 & 2 & 1.44 & 0.38 & 0.30 & - & & \\
\hline Pmac84 & 15 & 1 & 1.00 & 0.00 & 0.00 & - & & \\
\hline Pmac108 & 13 & 1 & 1.00 & 0.00 & 0.00 & - & & \\
\hline Pmac146 & 12 & 5 & 1.71 & 0.17 & 0.42 & ++ & & \\
\hline$m E g R 02$ & 14 & 1 & 1.00 & 0.00 & 0.00 & - & & \\
\hline$m A b R 28$ & 11 & 1 & 1.00 & 0.00 & 0.00 & - & & \\
\hline$m E g R 76$ & 14 & 2 & 1.51 & 0.00 & 0.34 & ++ & & \\
\hline$m E g R 78$ & 16 & 2 & 2.00 & 1.00 & 0.50 & - & & \\
\hline Média & 13.88 & 1.88 & 1.33 & 0.19 & 0.19 & & & \\
\hline
\end{tabular}




\begin{tabular}{|c|c|c|c|c|c|c|c|c|}
\hline Locus & $\mathrm{N}$ & $\mathrm{Na}$ & $\mathrm{Ne}$ & $\mathrm{Ho}$ & $\mathrm{He}$ & Null Allele & $F_{I S}$ & $p$-value \\
\hline Population S88 & & & & & & & 0.24 & 0.01 \\
\hline Pmac82 & 10 & 5 & 3.13 & 0.40 & 0.68 & ++ & & \\
\hline Pmac84 & 10 & 2 & 1.60 & 0.10 & 0.38 & - & & \\
\hline Pmac108 & 8 & 6 & 4.74 & 0.50 & 0.79 & ++ & & \\
\hline Pmac146 & 9 & 4 & 2.00 & 0.56 & 0.50 & - & & \\
\hline$m E g R 02$ & 7 & 1 & 1.00 & 0.00 & 0.00 & - & & \\
\hline$m A b R 28$ & 5 & 1 & 1.00 & 0.00 & 0.00 & - & & \\
\hline$m E g R 76$ & 10 & 1 & 1.00 & 0.00 & 0.00 & - & & \\
\hline$m E g R 78$ & 9 & 4 & 3.00 & 0.89 & 0.67 & - & & \\
\hline Média & 8.50 & 3.00 & 2.18 & 0.31 & 0.38 & - & & \\
\hline Population S115D & & & & & & & 0.59 & 1.00 \\
\hline Pmac82 & 19 & 2 & 1.05 & 0.05 & 0.05 & - & & \\
\hline Pmac84 & 19 & 2 & 1.70 & 0.58 & 0.41 & - & & \\
\hline Pmac108 & 19 & 2 & 1.98 & 0.89 & 0.49 & - & & \\
\hline Pmac146 & 18 & 6 & 3.27 & 1.00 & 0.69 & - & & \\
\hline$m E g R 02$ & 18 & 2 & 1.74 & 0.61 & 0.42 & - & & \\
\hline$m A b R 28$ & 15 & 1 & 1.00 & 0.00 & 0.00 & - & & \\
\hline$m E g R 76$ & 17 & 1 & 1.00 & 0.00 & 0.00 & - & & \\
\hline$m E g R 78$ & 18 & 2 & 2.00 & 1.00 & 0.50 & - & & \\
\hline Média & 17.88 & 2.25 & 1.72 & 0.52 & 0.32 & - & & \\
\hline Population S104 & & & & & & & 0.18 & 0.99 \\
\hline Pmac82 & 20 & 4 & 1.60 & 0.45 & 0.37 & - & & \\
\hline Pmac84 & 20 & 2 & 1.72 & 0.60 & 0.42 & - & & \\
\hline Pmac108 & 20 & 6 & 3.15 & 1.00 & 0.68 & - & & \\
\hline Pmac146 & 18 & 5 & 2.19 & 0.61 & 0.54 & - & & \\
\hline$m E g R 02$ & 20 & 1 & 1.00 & 0.00 & 0.00 & - & & \\
\hline$m A b R 28$ & 20 & 2 & 1.66 & 0.25 & 0.40 & - & & \\
\hline$m E g R 76$ & 20 & 1 & 1.00 & 0.00 & 0.00 & - & & \\
\hline$m E g R 78$ & 19 & 1 & 1.00 & 0.00 & 0.00 & - & & \\
\hline Média & 19.63 & 2.75 & 1.67 & 0.36 & 0.30 & - & & \\
\hline Population S114 & & & & & & & 0.01 & 0.52 \\
\hline Pmac82 & 20 & 2 & 1.05 & 0.05 & 0.05 & - & & \\
\hline Pmac84 & 20 & 4 & 2.02 & 0.55 & 0.50 & - & & \\
\hline Pmac108 & 19 & 4 & 2.06 & 0.68 & 0.52 & - & & \\
\hline Pmac146 & 13 & 2 & 1.83 & 0.54 & 0.45 & - & & \\
\hline$m E g R 02$ & 20 & 1 & 1.00 & 0.00 & 0.00 & - & & \\
\hline$m A b R 28$ & 20 & 1 & 1.00 & 0.00 & 0.00 & - & & \\
\hline$m E g R 76$ & 20 & 1 & 1.00 & 0.00 & 0.00 & - & & \\
\hline$m E g R 78$ & 15 & 2 & 1.92 & 0.27 & 0.48 & - & & \\
\hline Média & 18.38 & 2.13 & 1.48 & 0.26 & 0.25 & & & \\
\hline
\end{tabular}

$p<0.01 / 0.001=$ significant, and $p>0.01 / 0.001=$ not significant.

leles were found in populations S113, S88, S115D, S114, S104 and S82/83 (Table S4). The LD analysis results between polymorphic loci were not statistically significant after Bonferroni correction.

The FreeNA-corrected estimate of global $F_{\mathrm{ST}}$ was 0.44, ranging from 0.12 (Pmac82) to 0.79 ( $m E g R ~ 02)$ (Table S5). Clustering analyses (NJ, PCoA, STRUCTURE) have shown somewhat distinct results (Figure 1 and Figure $\mathrm{S} 1)$. However, the results from AMOVA suggest that the three clusters recovered by STRUCTURE better explain the genetic variation structuring in our data (Table 3), as follows: 1) S113, S014 and S115D populations; 2) S80,
S88 and S114 populations; 3) S82/S83 (Figure 1a). To investigate sub-structuration within our data, we performed a STRUCTURE analysis for each cluster, which resulted in each location being a cohesive genetic group (Figure 1b). Although we have not done an extensive geographic sampling for each studied taxon, and the number of markers is relatively low, some clustering results recovered here agree with previous phylogeographic hypotheses established for C. fernambucensis and C. insularis based on cpDNA and the PHYC gene (Franco et al., 2017b). The close relationship of C. jamacaru and C. fernambucensis subsp. fernambucensis (S104) deserves additional investigation, 
a
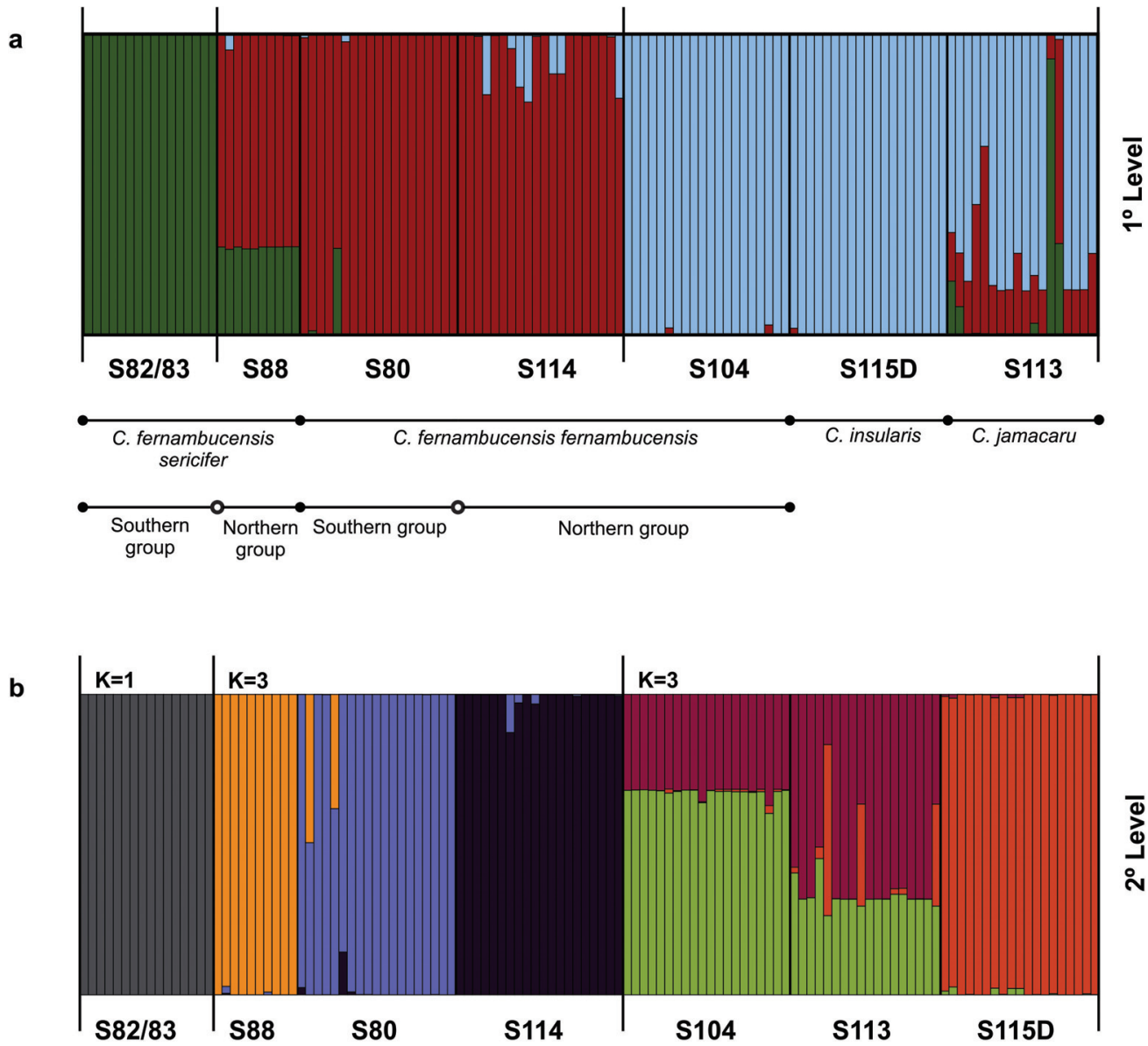

Figure 1 - Population differentiation in STRUCTURE, (a) results for $\mathrm{K}=3$ on the first level structure, and (b) separating each population as a distinct genetic group. The southern and northern population groups of C. fernambucensis subsp. sericifer and C. fernambucensis subsp. fernambucensis are based on phylogeographic circumscription (Franco et al., 2017b).

Table 3 - Global and hierarchical Analysis of Molecular Variance (AMOVA). For hierarchical AMOVA the a priori groups are based on taxonomic circumscription, NJ phenogram, PCoA and STRUCTURE.

\begin{tabular}{|c|c|c|c|}
\hline Groups & Fixation Indexes & Source of Variation & Percentege of Variation \\
\hline \multicolumn{4}{|l|}{ Global AMOVA } \\
\hline All populations only one group & $\Phi_{\mathrm{ST}}=0.34460$ & $\begin{array}{l}\text { Among populations } \\
\text { within group }\end{array}$ & 34.46 \\
\hline \multicolumn{4}{|l|}{ Taxonomic circumscription } \\
\hline \multirow[t]{2}{*}{ S113, S115D, S82/S83 with S88, S80 with S104 and S114 } & $\Phi_{\mathrm{CT}}=0.01385$ & Among groups & 1.38 \\
\hline & $\Phi_{\mathrm{SC}}=0.33731$ & $\begin{array}{l}\text { Among populations } \\
\text { within groups }\end{array}$ & 33.26 \\
\hline \multicolumn{4}{|l|}{ NJ Phenogram } \\
\hline \multirow[t]{2}{*}{ S113, S80 with S82/83 and S88, S104 with S115D, S114 } & $\Phi_{\mathrm{CT}}=-0.25331$ & Among groups & -25.33 \\
\hline & $\Phi_{\mathrm{SC}}=0.45546$ & $\begin{array}{l}\text { Among populations } \\
\text { within groups }\end{array}$ & 57.08 \\
\hline \multicolumn{4}{|l|}{ PCoA } \\
\hline & $\Phi_{\mathrm{CT}}=-0.26648$ & Among groups & -26.65 \\
\hline S113, all others populations (S80, S82/83, S88, S104, S114 and S115D) & $\Phi_{\mathrm{SC}}=0.38531$ & $\begin{array}{l}\text { Among populations } \\
\text { within groups }\end{array}$ & 48.80 \\
\hline \multicolumn{4}{|l|}{ STRUCTURE } \\
\hline \multirow[t]{2}{*}{ S82/S83, S80 with S88 and S114, S104 with S113 and S115D } & $\Phi_{\mathrm{CT}}=0.34737$ & Among groups & 34.74 \\
\hline & $\Phi_{\mathrm{SC}}=0.09764$ & $\begin{array}{l}\text { Among populations } \\
\text { within groups }\end{array}$ & 6.37 \\
\hline
\end{tabular}


but seems to be a spurious grouping as a result of the reduced number of sampled populations.

The estimated success in transferability observed in this study (35.16\%) was higher than the average of $10 \%$ found in cross-genera transferability studies published between 1997 and mid-2006 (see Barbará et al., 2007). However, this is not an uncommon result, as similar findings or even higher levels of cross-genera transferability were observed in some groups of plants (Satya et al., 2016). In the family Iridaceae, for example, a success of $77 \%$ was observed in cross-amplification between genera (Miz et al., 2016). In the family Malvaceae cross-genera SSR transferability varied from $71 \%$ to $92 \%$ (Satya et al., 2016), while in Euphorbiaceae these percentages ranged from $9.5 \%$ to $59.1 \%$ (Whankaew et al., 2011). Evidently, genera are taxonomic categories mainly based on morphological instead of genetic information, and different levels of phylogenetic divergence must be embedded within each genus. Therefore, the success in cross-genera transferability may vary highly depending on the target organism. On the other hand, it is expected that the success in cross-amplification should be a function of phylogenetic distance, at least regarding genetic differentiation (Barbará et al., 2007).

This expectation was not clearly observed here considering cactus phylogeny (Hernández-Hernández et al., 2014). We observed similar success in heterologous amplification using primers described for relatively distantly $(A$. bravoanus and E. grusonii - four transferred of nine tested) or closely related species ( $P$. machrisii - five transferred of 11 tested). It is worth highlighting that for those loci from $A$. bravoanus, E. grusonii, and $P$. chichipe we had a previous expectation of positive cross-amplification, as they were formerly transferred for some Cereus species (Martin, 2011; Fernandes et al., 2016). Nevertheless, higher levels of cross-genera amplification in the cactus family might be a widespread tendency, as the main lineage divergences and species radiation events within this family are thought to have occurred in the last $10 \mathrm{Myr}$ (Arakaki et al., 2011; Hernández-Hernández et al., 2014; Silva et al., 2018). Even with remarkable morphological distinctness among cactus species, resulting in more than 120 recognized genera (Hunt et al., 2006), these recent divergence times increase the possibility of heterologous amplification in the family Cactaceae due to the expected similarities in the flanking SSR regions among different species. Evidently, this is not a rule, as even here we found discordance between some results obtained by Martin (2011) and Fernandes et al. (2016), which were likely due to nucleotide differences in flanking regions of the distinct samples. At any rate, this information should be taken into consideration to encourage cross-genera transferability studies in Cactaceae, which, despite their potential, are still relatively scarce in this family (Table S6).

The genus Cereus constitutes an interesting biological model to perform evolutionary studies, and efforts were employed to screen informative molecular markers in this genus (Silva et al., 2016; Romeiro-Brito et al., 2016) to solve species level phylogeny (Franco et al., 2017a) and to investigate population differentiation and phylogeography (Franco et al., 2017b; Silva et al., 2018). Our results are in line with these endeavors, supplying additional molecular markers that can be useful for estimating genetic diversity and gene flow in target Cereus species. Furthermore, considering the rate of success in transferability, our results should encourage cactus researchers interested in using the increasing number of SSR loci that have been described for representatives of this highly diverse and relatively overlooked plant family (e.g., Bonatelli et al., 2015; Fava et al., 2016).

\section{Acknowledgments}

This study was financed in part by the Coordenação de Aperfeiçoamento de Pessoal de Nível Superior - Brasil (CAPES) - Finance Code 001 (fellowship to J.R.B.). This work was also supported by grants from São Paulo Research Foundation (FAPESP) to F.F.F. (2010/25227-0, 2014/25227-0). To sampling Cereus insularis, we had support from Fernando de Noronha Marine National Park (ICMBIO/PARNAMAR) and governmental administration of Fernando de Noronha (DEFN). We thank Maria de Fatima P.S. Machado (State University of Maringá, Brazil) for supplying valuable information about SSR transferability in Cereus. We thank Manolo F. Perez for critical comments in a preliminary version of this work.

\section{Conflict of interest}

The authors declare that they have no conflict of interest associated with this study.

\section{Author contributions}

F.F.F. and J.R.B. conceived and designed the study; J.R.B. conducted the experiments; J.R.B., I.A.S.B and G.A.R.S. analyzed the data; F.F.F. and J.R.B. wrote the manuscript; E.M.M, D.C.Z. and N.P.T contributed in data interpretation, writing and grammar review, all authors read and approved the final version.

\section{References}

Albert S and Schmitz J (2002) Characterization of major royal jelly protein-like DNA sequences in Apis dorsata. J Apic Res 41:75-82.

Arakaki M, Christin PA, Nyffeler R, Lendel A, Eggli U, Ogburn RM, Spriggs E, Moore MJ and Edwards EJ (2011) Contemporaneous and recent radiations of the world's major succulent plant lineages. Proc Natl Acad Sci U S A 108:8379-8384.

Barbará T, Palma-Silva C, Paggi GM, Bered F, Fay MF and Lexer C (2007) Cross-species transfer of nuclear microsatellite markers: potential and limitations. Mol Ecol 16:3759-3767. 
Beatty GE and Provan J (2011) Comparative phylogeography of two related plant species with overlapping ranges in Europe, and the potential effects of climate change on their intraspecific genetic diversity. BMC Evol Biol 11: 29

Bonatelli IAS, Carstens BC and Moraes EM (2015) Using Next Generation RAD sequencing to isolate multispecies microsatellites for Pilosocereus (Cactaceae). PLoSOne 10: e0142602.

Cavalli-Sforza LL and Edwards AWF (1967) Phylogenetic analysis: Models and estimation procedures. Am J Hum Genet 19:233-257.

Chapuis MP and Estoup A (2007) Microsatellite null alleles and estimation of population differentiation. Mol Biol Evol 24:621-631.

Don RH, Cox PT, Wainwright BJ, Baker K and Mattick JS (1991) Touchdown PCR to circumvent spurious priming during gene amplification. Nucleic Acids Res 19:4008.

Evanno G, Regnault S and Goudet J (2005) Detecting the number of clusters of individuals using the software STRUCTURE: A simulation study. Mol Ecol 14:2611-2620.

Excoffier L and Lischer HE (2010) Arlequin suite ver 3.5: A new series of programs to perform population genetics analyses under Linux and Windows. Mol Ecol Resour 3:564-7.

Fava WS, Paggi GM, Zanella CM and Lorenz-Lemke AP (2016) Development and characterization of microsatellite markers for Echinopsis rhodotricha and cross-amplification in other species of Cactaceae. Biochem Syst Ecol 66:19-23.

Franco FF, Rodrigues GAS, Marsola EM, Taylor NP, Zappi DC, Jojima CL and Machado MC (2017a) Plio-Pleistocene diversification of Cereus (Cactaceae, Cereeae) and closely allied genera. Bot J Linn Soc 183:199-210.

Franco FF, Jojima CL, Perez MF, Zappi DC, Taylor NP and Moraes EM (2017b) The xeric side of the Brazilian Atlantic Forest: The forces shaping phylogeographic structure of cacti. Ecol Evol 7:9281-9293.

Fernandes VNA, Neves AF, Martin PG, Mangolin CA and Machado MFPS (2016) Genetic structure and molecular divergence among samples of mandacaru (Cereus spp.; Cactaceae) as revealed by microsatellite markers. Biochem Syst Ecol 64:38-45.

Goldstein DB and Pollock DD (1997) Lauching microsatellites: A review of mutation processes and methods of phylogenetic interference. J Hered 88:335-342.

Gómez-Fernández A, Alcocer I and Matesanz S (2016) Does higher connectivity lead to higher genetic diversity? Effects of habitat fragmentation on genetic variation and population structure in a gypsophile. Conserv Genet 17:631-641.

Goudet J (1995) FSTAT (version 1.2): A computer program to calculate F-statistics. J Hered 86:485-486.

Haerinasab M, Rahiminejad MR and Ellison NW (2016) Transferability of Simple Sequence Repeat (SSR) markers developed in red clover (Trifolium pretense L.) to some Trifolium species. Iran J Sci Technol Trans Sci 40:59-62.

Hardesty BD, Hughes L, Rodriguez VM and Hawkins JA (2008) Characterization of microsatellite loci for the endangered cactus Echinocactus grusonii, and their cross-species utilization. Mol Ecol Res 8:164-167.

Hernández-Hernández T, Brown JW, Schlumpberger BO, Eguiarte LE and Magallón S (2014) Beyond aridification: Multiple explanations for the elevated diversification of cacti in the New World succulent biome. New Phytol 202:1382-1397.
Hughes SL, Rodriguez VM, Hardesty BD, Luna RTB, Hernández HM, Robson RM and Hawkins JA (2008) Characterization of microsatellite loci for the critically endangered cactus Ariocarpus bravoanus. Mol Ecol Res 8:1068-1070.

Hunt D, Taylor N and Charles G (2006) The new cactus lexicon. DH Books, Milborne Port, 526 pp.

Jakobsson M and Rosenberg NA (2007) CLUMPP: A cluster matching and permutation program for dealing with label switching and multimodality in analysis of population structure. Bioinformatics 23:1801-1806.

Jan C, Dawson DA, Altringham JD, Burke T and Butlin RK (2012) Development of conserved microsatellite markers of high cross-species utility in bat species (Vespertilionidae, Chiroptera, Mammalia). Mol Ecol 12:532-548.

Lavor P, Van Den Berg C and Versieux LM (2013) Transferability of 10 nuclear microsatellite primers to Vriesea minarum (Bromeliaceae), a narrowly endemic and threatened species from Brazil. Braz J Bot 36:165-168.

Martin PG (2011) Transferibilidade de microssatélites de cactáceas para a análise de regenerantes clonais (R0) de Cereus peruvianus Mill. (Cactaceae). M. Sc. Thesis, Universidade Estadual de Maringá, Maringá.

Mehmood A, Luo S, Ahmad NM, Dong C, Mahmood T, Sajjad Y, Jaskani MJ and Sharp P (2016) Molecular variability and phylogenetic relationships of guava (Psidium guajava L.) cultivars using inter-primer binding site (iPBS) and microsatellite (SSR) markers. Genet Resour Crop Evol 63:1345-1361.

Mengistu FG, Motoike SY, Caixeta ET, Cruz CD and Kuki KN (2016) Cross-species amplification and characterization of new microsatellite markers for the macaw palm, Acrocomia aculeata (Arecaceae). Plant Genet Resour 14:163-172.

Miz RB, Tacuatiá LO, Cidade FW, de Souza AP, Bered F, Eggers L and de Souza-Chies TT (2016) Isolation and characterization of microsatellite loci in Sisyrinchium (Iridaceae) and cross amplification in other genera. Genet Mol Res 15:38474

Moodley Y, Masello JF, Cole TL, Calderon L, Munimanda GK, Thali MR, Alderman R, Cuthbert RJ, Marin M, Massaro M et al. (2015) Evolutionary factors affecting the cross-species utility of newly developed microsatellite markers in seabirds. Mol Ecol 15:1046-1058.

Nogueira AM, Ferreira A and Ferreira MFS (2015) Transferability of microsatellites from Psidium guajava to Eugenia, Myrciaria, Campomanesia, and Syzygium Species (Myrtaceae). Plant Mol Biol 34:249-256.

Oliveira EJ, Pádua JG, Zucchi MI, Vencovsky R and Vieira MLC (2006) Origin, evolution and genome distribution of microsatellites. Genet Mol Biol 29:294-307.

Otero-Arnaiz A, Cruse-Sanders J, Casas A and Hamrick JL (2004) Isolation and characterization of microsatellites in the columnar cactus: Polaskia chichipe and cross-species amplification within the Tribe Pachycereeae (Cactaceae). Mol Ecol Notes 4:265-267.

Palma-Silva C, Wendt T, Pinheiro F, Barbará T, Fay MF, Cozzolino S and Lexer C (2011) Sympatric bromeliad species (Pitcairnia spp.) facilitate tests of mechanisms involved in species cohesion and reproductive isolation in Neotropical inselbergs. Mol Ecol 20:3185-3201. 
Peakall R and Smouse PE (2012) GENALEX 6: Genetic analysis in Excel. Population genetic software for teaching and research. Mol Ecol Notes 6:288-295.

Perez MF, Téo MF, Zappi DC, Taylor NP and Moraes EM (2011) Isolation, characterization, and cross-species amplification of polymorphic microsatellite markers for Pilosocereus machrisii (Cactaceae). Am J Bot 98:204-206.

Pinheiro F, Cozzolino S, Draper D, Barros F, Félix LP, Fay MF and Palma-Silva C (2014) Rock outcrop orchids reveal the genetic connectivity and diversity of inselbergs of northeastern Brazil. BMC Evol Biol 14:1471-2148.

Pritchard JK, Stephens M and Donnelly P (2000) Inference of population structure using multilocus genotype data. Genet Soc Am 155:945-959.

Ribeiro PCC, Muller LAC, Lemos-Filho JP and Lovato MB (2014) Transferability and characterization of nuclear microsatellite markers in populations of Annona coriacea (Annonaceae), a tree from the Brazilian Cerrado. Bot Soc Sao Paulo 37:353-356.

Rice WR (1989) Analyzing tables of statistical tests. Evol 43:223-225.

Romeiro-Brito M, Moraes EM, Taylor NP, Zappi DC and Franco FF (2016) Lineage-specific evolutionary rate in plants: Contributions of a screening for Cereus (Cactaceae). Applic Plant Sci 4:1500074.

Rosenberg NA (2004) DISTRUCT: A program for the graphical display of population structure. Mol Ecol Notes 4:137-138.

Saitou N and Nei M (1987) The neighbor-joining method: a new method for reconstructing phylogenetic trees. Mol Biol Evol 4:406-425.

Satya P, Paswan PK, Ghosh S, Majumdar S and Ali N (2016) Confamiliar transferability of simple sequence repeat (SSR) markers from cotton (Gossypium hirsutum L.) and jute (Corchorus olitorius L.) to twenty two Malvaceous species. Biotech 6:65-70.

Silva GAR, Jojima CL, Moraes EM, Antonelli A, Manfrin MH and Franco FF (2016) Intra and interspecific sequence variation in closely related species of Cereus (CACTACEAE). Biochem Syst Ecol 65:137-142.

Silva GAR, Antonelli A, Lendel A, Moraes EM and Manfrin MH (2018) The impact of early Quaternary climate change on the diversification and population dynamics of a South American cactus species. J Biogeogr 45:76-88.

Tan LQ, Wang LY, Xu LY, Wu LY, Peng M, Zhang CC, Wei K, Bai PX, Li HL, Cheng H and Qi GN (2016) SSR-based genetic mapping and QTL analysis for timing of spring bud flush, young shoot color, and mature leaf size in tea plant (Camellia sinensis). Tree Genet Genomes 12:52-64.

Van Oosterhout C, Hutchinson WF, Wills DPM and Shipley P (2004) MICRO-CHECKER: Software for identifying and correcting genotyping errors in microsatellite data. Mol Ecol Notes 4:535-538.

Whankaew S, Kanjanawattanawong S, Phumichai C, Smith DR, Narangajavana J and Triwitayakor K (2011) Cross-genera transferability of (simple sequence repeat) SSR markers among cassava (Manihot esculenta Crantz), rubber tree (Hevea brasiliensis Muell. Arg.) and physic nut (Jatropha curcas L.). Afr J Biotechnol 10:1768-1776.

Weir BS and Cockerham CC (1984) Estimating F-statistics for the analysis of population-structure. Evolution 38:1358-1370.

Zhu XH, Cheng SP, Liao T and Kang XY (2016) Genetic diversity in fragmented populations of Populus talassica inferred from microsatellites: Implications for conservation. Genet Mol Res 15:27899.

\section{Internet Resources}

Structure Harvester, http://taylor0.biology.ucla.edu/structureHarvester/ (accessed 10 August 2016)

Langella O (1999) Populations 1.2.32, http://bioinformatics.org/ tryphon/populations/ (accessed 05 August 2016)

\section{Supplementary material}

The following online material is available for this article:

Figure S1 - Neighbor-joining phenogram.

Table S1 - Characteristics of 20 microsatellite loci tested for transferability.

Table S2 - Transferability results of the SSR markers for populations.

Table S3 - PCR conditions for the SSR markers transferred. Table S4 - Private alleles ( $>10 \%$ frequency) found in each locus per population.

Table S5 - F-statistics by locus for all populations and FST corrected by ENA method.

Table S6 - Results of a literature survey.

Associate Editor: Dario Grattapaglia

License information: This is an open-access article distributed under the terms of the Creative Commons Attribution License (type CC-BY), which permits unrestricted use, distribution and reproduction in any medium, provided the original article is properly cited. 